\title{
JiStar: Reconciliando Código Java com Modelagem Orientada a Metas
}

\author{
Ana Maria da Mota Moura ${ }^{1}$ e Julio Cesar Sampaio do Prado Leite ${ }^{1}$ \\ ${ }^{1}$ Departamento de Informática - Pontifícia Universidade Católica do Rio de Janeiro \\ Rio de Janeiro - RJ - Brasil \\ \{ammmoura, julio\}@inf.puc-rio.br
}

\begin{abstract}
Once a software has been built without the goal-oriented requirements engineering perspective, how can you understand your goals for evolutionary purposes? This work is an extension of our proposal for a backward traceability model between Java code and the $i *$ goal model through the use of code metadata. In the evolution of the JiStar framework, we reviewed the set of Java code annotations for $i$ * elements; we added the export in iStarML format, and; we enable the automatic or semi-automatic generation of goal models. We evaluated the feasibility of our framework on a real system called RioBus and the results show the suitability of this traceability for evolution purposes.
\end{abstract}

Resumo. Uma vez que um software tenha sido construido sem a perspectiva da engenharia de requisitos orientada a metas, como compreender suas metas para fins de evolução? Este trabalho é uma extensão da nossa proposta de modelo de rastreabilidade para trás entre código Java e o modelo de metas $i^{*}$ através do uso de metadados de código. Na evolução do framework JiStar, revisamos o conjunto de anotações de código Java para elementos do $i^{*}$; adicionamos a exportação no formato iStarML, e; possibilitamos a geração automática ou semiautomática de modelos de metas. Avaliamos a viabilidade do nosso framework sobre um sistema real chamado RioBus e os resultados indicam a adequação dessa rastreabilidade para fins de evolução.

\section{Introdução}

Este trabalho é uma extensão da proposta do framework JiStar 1.0 [Moura and Leite 2020] apresentado no WER 2020. Um dos alicerces da nossa pesquisa é a Engenharia de Requisitos Orientada a Metas (GORE - Goal Oriented Requirements Engineering) que, apesar de ser muito utilizada nas iterações iniciais do desenvolvimento de software [Darwish and Zohdy 2016; Yu 1995, 2001], já possui pesquisas que têm demonstrado a utilidade de modelos de metas durante o projeto de arquitetura, codificação, teste, monitoramento, adaptação e evolução [Bresciani et al. 2004; Horkoff et al. 2015]. GORE auxilia o engenheiro de requisitos a lidar com os interessados para descobrir suposições e fundamentos ocultos, assim como, desenvolver e explorar potenciais alternativas [Yu 2001] pois as metas capturam, em diferentes níveis de abstração, os

Cadernos do IME - Série Informática

e-ISSN: 2317-2193 (online)

DOI: $10.12957 /$ cadinf.2020.57030 
vários objetivos que o software deve alcançar [Yu 1995]. Algumas pesquisas têm feito o uso de GORE para além do desenvolvimento baseado em agentes, como integração com métodos ágeis [Jaqueira et al. 2013] e aplicações Web [Bolchini and Paolini 2002]. Deste modo, há indícios de que softwares construídos com o uso de GORE podem estar mais alinhados com as metas da organização e serem mais amenos para evolução. Neste contexto, como reconciliar softwares construídos sob outras perspectivas e/ou paradigmas com suas metas para fins de evolução?

Quando o software não foi desenvolvido sob a perspectiva GORE, boa parte do conhecimento sobre as metas (i.e.: razões da existência do software) fica disperso em emails, atas de reunião e sob a forma de conhecimento tácito dos envolvidos no projeto. Neste contexto, após algum tempo, não é trivial identificar se todas as metas já estão implementadas, ou reutilizar algumas operacionalizações de metas flexíveis, ou identificar o propósito de algumas partes do código com relação aos objetivos da organização, entre outros problemas de engenharia de software.

Este trabalho, assim como o trabalho [Moura and Leite 2020], é parte de uma pesquisa para a reengenharia de sistemas autoadaptativos guiada pelo requisito não funcional de consciência [Moura 2017], onde propomos um conjunto de heurísticas para extrair um modelo de metas a partir de sistemas orientados a objetos (OO) a fim de evoluir tais sistemas com base em suas metas flexíveis de consciência ainda que não tenham sido construídos sob a perspectiva GORE. Alguns trabalhos na literatura sugerem abordagens para o mapeamento do modelo de metas até a implementação do sistema [Alencar et al. 2003; Bresciani et al. 2004; Serrano 2011], ou seja, visam garantir que o software construído corresponda ao desejado [Silva et al. 2004], gerando vínculos os quais chamamos de rastreabilidade [Almentero 2013; Sayão and Do Prado Leite 2006; Silva et al. 2004]. A rastreabilidade pode ser avante (dos requisitos para o código) e/ou para trás (do código para os requisitos). Esta última poderia ser utilizada para gerar um modelo intencional a partir do código, porém softwares desenvolvidos com outros paradigmas não orientados a agentes (ex.: Programação Orientado a Objetos - POO) podem gerar modelos de meta extensos e pouco abstratos, conforme observamos ao aplicarmos as nossas heurísticas em estudos de caso com sistemas reais para diferentes finalidades de reengenharia [Moura et al. 2019; Oliveira et al. 2018]. Apesar das nossas heurísticas possibilitarem uma forma semiautomatizada de gerar modelos de metas $i^{*}$, nestes estudos, nos deparamos com modelos de metas extensos que foram utilizados com alguma dificuldade pelos pesquisadores que não eram familiarizados com o código, devido à nomenclatura e quantidade dos elementos que vinham diretamente do código fonte.

Diante deste contexto, a principal questão de pesquisa neste trabalho, assim como no trabalho [Moura and Leite 2020], é: Como obter um modelo de metas a partir de um código que foi construído sem a perspectiva GORE, com um nível de abstração mais próximo dos objetivos da aplicação, sem perder a rastreabilidade com o código? Porém, neste trabalho mostramos as evoluções realizadas no framework JiStar baseadas nos trabalhos futuros previstos em [Moura and Leite 2020] e em atendimento a nossa pesquisa principal para a reengenharia de sistemas conscientes .

O escopo da nossa pesquisa é delimitado ao uso do framework i* [Yu 1995], para representar o modelo de metas, e ao paradigma OO, pois o nosso conjunto de heurísticas para a rastreabilidade para trás [Moura 2017] considera características 
específicas de programação orientada a objetos (POO). O i* foi escolhido por ser um framework bem difundido na literatura [Dalpiaz et al. 2016b] e com inúmeras extensões $i^{*}$ (vide catálogo ${ }^{1}$ [Gonçalves et al. 2018]).

Em resposta à nossa questão de pesquisa, definimos um modelo de rastreabilidade para trás, do código fonte para o modelo de metas da aplicação, através do uso de metadados de código. Esta rastreabilidade pode prover a geração automática de modelos de metas a partir do código anotado e, a partir desta nova versão, também pode gerar modelos de metas $i^{*}$ com base em nosso conjunto de heurísticas [Moura 2017]. Em particular, entre modelos em i* e sistemas desenvolvidos em Java. A linguagem Java foi escolhida por sua ampla utilização na indústria e na academia. A rastreabilidade é proposta através de um framework denominado $\mathrm{JiStar}^{2}$, o qual é composto por um conjunto de anotações de código Java, para representar as informações dos modelos $i^{*}$, onde este conjunto foi revisado nesta nova versão, e um gerador de modelo de metas em HTML, PiStar 2.0 [Pimentel and Castro 2018] que agora também exporta para iStarML [Cares et al. 2007].

Neste trabalho revisitaremos a avaliação feita em [Moura and Leite 2020], onde a avaliação de viabilidade da nossa abordagem usando o código fonte de um aplicativo chamado RioBus visando reconciliá-lo com as metas as quais seus usuários conseguem alcançar em sua versão atual.

A partir desta introdução, o restante do artigo está organizado da seguinte forma: $\mathrm{Na}$ Seção 2, apresentamos a fundamentação teórica. Na Seção 3, relacionamos alguns trabalhos correlatos. Na Seção 4, apresentamos o conjunto de anotações revisado do framework JiStar, enquanto na Seção 5 mostramos a exportação de modelos de metas para HTML, PiStar 2.0 e iStarML utilizando a nova versão do JiStar. Na Seção 6, apresentamos nosso estudo de viabilidade com base no aplicativo móvel real denominado RioBus. Finalmente, na Seção 7, apresentamos nossas conclusões ressaltando as contribuições, desafios e trabalhos futuros.

\section{Fundamentação Teórica}

\subsection{Framework $i^{*}$}

A versão do framework $i^{*}$ escolhida para esta pesquisa é a 1.0 [Yu 1995], porque a sua intencionalidade distribuída auxilia a modularização, e a relação meios-fim é o ponto chave para mapear a variabilidade. Não utilizamos a versão iStar 2.0 [Dalpiaz et al. 2016a] pois ela não permite indicar o meio para alcançar um recurso e o relacionamento refinamento, que permite refinar metas em tarefas e vice-versa, possui semântica diferente do relacionamento meios fim. Além disso, a riqueza presente nos tipos de relacionamentos da versão 1.0 nos ajuda a qualificar melhor as relações existentes entre os elementos.

O framework $i^{*}$ define meta como uma condição ou estado de algo no mundo que o ator gostaria de alcançar [Yu 1995]. O framework $i^{*}$ consiste de dois modelos: O modelo de Dependência Estratégica (SD - Strategic Dependency) que descreve o

\footnotetext{
${ }^{1}$ http://istarextensions.cin.ufpe.br/catalogue/.

${ }^{2}$ https://github.com/RE-Projects/JiStar
} 
processo em termos de relacionamentos de dependência intencional entre agentes que dependem um do outro para que metas sejam alcançadas, tarefas sejam executadas, recursos sejam fornecidos ou metas-flexíveis sejam satisfeitas a contento; e o modelo de Raciocínio Estratégico (SR - Strategic Rationale) que descreve as questões e preocupações que os agentes têm acerca de processos existentes e alternativas propostas, e como eles podem ser endereçados em termos de uma rede de relacionamentos means-end.

Os elementos do modelo SD do framework i* são: o Ator (Actor) que, em geral, é uma unidade para a qual dependências intencionais podem ser descritas. Este pode ser especializado em três tipos a saber: Agentes (Agents), Papéis (Roles) e Posições (Positions). Um Agente é um ator com manifestações físicas e concretas, como um indivíduo, mas pode ser usado para referir-se a humanos assim como agentes artificiais (hardware/software). Um Papel é uma caracterização abstrata do comportamento de um ator social dentro de um contexto especializado ou domínio. Uma Posição é uma abstração intermediária entre um papel e um agente. Os atores possuem relacionamento específicos entre si: Um agente ocupa uma posição (occupies); mas também pode desempenhar um papel (plays); enquanto uma posição cobre (covers) vários papéis, e; todos eles são um tipo de (is-a) ator. Todos os tipos de ator podem ter subpartes (ispart-of). Todos os tipos de atores ainda podem ser uma instanciação de um ator mais genérico (ins). O relacionamento de Dependência (Dependency) estabelece uma relação entre dois atores onde um depende (depender) do outro (dependee) para alcançar alguma meta ou objetivo (dependum). Um dependum pode ser uma meta, uma metaflexível, uma tarefa ou um recurso. Cada um será explicado no modelo SR.

Os elementos do modelo SR do framework i* são: a Meta (Goal) uma condição ou estado de algo no mundo que o ator gostaria de alcançar, enquanto Meta-Flexível (Softgoal) é uma condição no mundo que o ator gostaria de alcançar, porém os critérios para que esta condição seja alcançada não são claramente definidos a princípio e estão sujeitos à interpretação. Geralmente é uma meta de qualidade que guia ou restringe os outros elementos. A Tarefa (Task) especifica um modo de fazer algo. Quando uma tarefa é especificada como um subcomponente de uma tarefa maior, isto restringe a tarefa maior àquele curso de ação. O Recurso (Resource) é uma entidade (física ou informacional). Finalmente, uma fronteira de ator (ator genérico, agente, papel ou posição) é usada como uma estrutura modular para representar o raciocínio interno de um ator.

\subsection{Mapeamento de Modelo $i^{*}$ para Sistemas OO}

$\mathrm{Na}$ literatura, encontramos trabalhos com propostas para integrar modelos de metas (onde são especificados requisitos organizacionais) e modelos orientados a objetos (OO). Em [Castro et al. 2000], os autores criaram um conjunto de regras de transformação de modelos $\mathrm{i}^{*}$ para modelos OO usando pUML (precise UML) em conjunto com OCL (Object Constraint Language). O conjunto é formado por seis regras gerais de transformação e esta transformação pode ser realizada com o apoio da extensão GOOD (Goals into Object Oriented Development) para a ferramenta Rational Rose. Esta extensão faz parte da ferramenta OME (Organization Modeling Environment). 
Em 2003, este conjunto de regras foi estendido [Alencar et al. 2003] e para suportá-lo foi desenvolvida uma versão estendida da ferramenta GOOD denominada XGOOD. Este conjunto de regras estendido (ver Tabela 1) foi publicado em livro no ano de 2011 [Castro et al. 2011] e em 2015 foi a base para o desenvolvimento de um novo ferramental de apoio. Neste novo ferramental de apoio, o modelo i* é desenvolvido na ferramenta iStarTool [Malta et al. 2011] e, posteriormente, exportado no formato XMI para que seja importado na ferramenta Eclipse (com um plugin da linguagem ATL). Em seguida, as regras de transformação descritas agora em ATL são aplicadas e o modelo OO de saída é gerado também no formato XMI, o que possibilita sua importação em uma ferramenta CASE para visualização do modelo resultante.

Se utilizássemos as heurísticas de mapeamento do modelo de metas para representações $O O$ (ver Tabela 1), tal como são descritas, no sentido para trás, então poderíamos gerar modelos extensos e menos abstratos. Além disso, alguns elementos da POO como interfaces e classes abstratas que, muitas vezes são utilizadas para tratar variabilidade, não teriam correspondência para mapeamento e seria difícil definir se uma classe deveria ser mapeada para um agente, papel ou posição (ver regra G1.1 na Tabela 1). Nosso trabalho difere deste por fazer o mapeamento no sentido do código OO para o modelo de metas $i^{*}$ através do uso de metadados de código, ou seja, nós identificamos para elementos da POO quais os elementos i* eles podem representar.

Tabela 1. Regras de transformação [Alencar et al. 2003; Castro et al. 2011].

\begin{tabular}{|c|c|c|}
\hline Regra & $\mathbf{i}^{*}$ & pUML \\
\hline G1.1 & Agentes, papéis ou posições & Classes \\
\hline G1.2 & Relacionamento is-part-of & Agregação \\
\hline G1.3 & Relacionamento is- $a$ & Generalização / especialização \\
\hline G1.4 & Relacionamento occupies & Associação nomeada occupies \\
\hline G1.5 & Relacionamento covers & Associação nomeada covers \\
\hline G1.6 & Relacionamento plays & Associação nomeada plays \\
\hline G2.1 & Tarefas no modelo SD & Métodos com visibilidade pública na classe fornecedora \\
\hline G2.2 & Tarefas no modelo SR & Métodos com visibilidade privada na classe fornecedora \\
\hline G3.1 & Recursos no modelo SD & $\begin{array}{l}\text { Classe, se a dependência tem característica de um } \\
\text { objeto, ou, caso contrário, atributo com visibilidade } \\
\text { privada na classe fornecedora }\end{array}$ \\
\hline G3.2 & Recurso (sub-recurso) no modelo SR & $\begin{array}{l}\text { Atributo com visibilidade privada na classe, se não } \\
\text { puder ser entendido como um objeto, ou, caso contrário, } \\
\text { uma classe }\end{array}$ \\
\hline G4.1 & Meta-Flexível no modelo SD & Atributo com visibilidade pública na classe fornecedora \\
\hline G4.2 & Meta-Flexível no modelo SR & $\begin{array}{l}\text { Atributo com visibilidade privada na classe à qual a } \\
\text { meta-flexível pertence }\end{array}$ \\
\hline G5 & Relacionamento task-decomposition & $\begin{array}{l}\text { Pré e pós condições em OCL na operação } \\
\text { correspondente }\end{array}$ \\
\hline G6.1 & $\begin{array}{l}\text { Relacionamento means-end de Meta- } \\
\text { flexível para Meta-Flexível }\end{array}$ & $\begin{array}{l}\text { A disjunção dos valores dos meios implica no valor do } \\
\text { fim }\end{array}$ \\
\hline G6.2 & $\begin{array}{l}\text { Relacionamento means-end de tarefa } \\
\text { para meta-flexível e tarefa para recurso }\end{array}$ & $\begin{array}{l}\text { A pós-condição das tarefas meio implicam no valor do } \\
\text { fim }\end{array}$ \\
\hline G6.3 & $\begin{array}{l}\text { Relacionamento means-end de tarefa } \\
\text { para tarefa }\end{array}$ & $\begin{array}{l}\text { A disjunção da pós-condição dos meios implica as pós- } \\
\text { condições do fim }\end{array}$ \\
\hline
\end{tabular}




\section{Trabalhos Relacionados}

$\mathrm{O}$ trabalho de $\mathrm{Yu}$ et. al. [Yu et al. 2005] propõe uma estratégia de engenharia reversa de sistemas legados com vistas a obter um modelo de metas para fins de reengenharia de sistemas legados que oferecem algum tipo de serviço em componentes e serviços que permitam reutilizar o serviço oferecido de diferentes formas. O referido trabalho recupera modelos de meta a partir de código legado estruturado (i.e.: OO) e não estruturado. De um modo geral, a técnica é composta por quatro passos principais: 1) refatorar o Código fonte através da extração de métodos considerando seus comentários de código; 2) converter o código refatorado em um programa estruturado e abstrato através de refatoração com statechart e da construção de gráficos de hammock; 3) extrair um modelo de metas a partir da árvore de sintaxe abstrata do programa estruturado; e 4) identificar NFR e derivar metas-flexíveis baseado na rastreabilidade entre o código e o modelo de metas e no uso de testes de função. Ou seja, os RNF são derivados a partir da observação dos efeitos nas métricas de qualidade ao se ligar/desligar os RNF identificados. A nossa estratégia [Moura and Leite 2020] se diferencia pelo uso de anotações de código para auxiliar na rastreabilidade dos elementos do POO (Programação Orientada a Objetos) para os elementos do modelo i*. Além disso, fazemos uso de fronteiras de atores (ver Seção 2.1) como uma estrutura modular, possibilitando representar elementos modulares do POO. É importante também destacar que, nesta nova versão, possibilitamos o uso de heurísticas [Moura 2017] para a geração de modelos de metas $i^{*}$ que podem ser utilizadas em conjunto com as anotações ou separadamente.

O trabalho [Serrano and Leite 2011] propõe o desenvolvimento de sistemas multiagentes centrados no conceito de intencionalidade. Os autores descrevem um conjunto de heurísticas para o mapeamento de modelos $i^{*}$ para código baseado em BDI (Belief - Desire - Intention) usando JADEX. Em sua Tese, Serrano [Serrano 2011] relata que o conjunto de heurísticas de mapeamento proposto poderia ser utilizado no sentido inverso para obter um modelo de metas $i^{*}$ a partir de código JADEX baseado em BDI, ou seja, uma rastreabilidade bidirecional. O nosso trabalho difere deste por possuir um conjunto de anotações e heurísticas que mapeiam sistemas OO para modelo $i^{*}$, possibilitando a rastreabilidade da intencionalidade de sistemas multiagentes construídos com linguagens $\mathrm{OO}$ e sistemas $\mathrm{OO}$ que não sejam multiagentes.

\section{JiStar - Conjunto de Anotações de Código}

O framework JiStar possibilita a rastreabilidade entre o código fonte Java e as metas do sistema, permitindo a geração automática e semiautomática de um modelo de metas em $i^{*}$. O framework possui um conjunto de anotações de código relacionadas aos elementos do framework $i^{*}$ para que o engenheiro de software possa fazer a correlação do sistema e suas metas. Com o uso deste framework é possível verificar quais metas estão sendo atendidas, tornar conhecidas as operacionalizações de metas flexíveis, realizar análise de impacto no caso de mudanças nas metas, entre outras vantagens advindas da rastreabilidade entre o código e o modelo de requisitos. A rastreabilidade é dada pelo uso dos nomes dos elementos no modelo que é oriundo das anotações de código ou do próprio código quando se utiliza unicamente as nossas heurísticas de mapeamento. 
Escrevemos um conjunto de anotações para manter a rastreabilidade de código Java para modelos de metas em i*, onde a aplicação de cada tipo de anotação foi inspirada nas nossas heurísticas [Moura 2017], mas permite enriquecer o mapeamento através de informações passadas nas propriedades das anotações. A vantagem de usar anotações isoladamente das heurísticas é que é possível anotar somente os elementos essenciais para a compreensão das metas organizacionais que são alcançadas por meio da aplicação, omitindo detalhes técnicos que poderiam deixar o modelo pouco abstrato e com um grande volume de informações. Porém o suo de heurísticas pode gerar um modelo i* sem o esforço de anota o código. Devido as vantagens de ambos formatos, criamos a possibilidade de exportar o modelo de metas com base em ambos considerando a prevalência das informações passadas através das anotações de código.

É importante destacar que todas as anotações foram são baseadas no uso de política de retenção runtime para estarem disponíveis em tempo de execução e poderem ser acessadas por reflexão. Todas as anotações também foram especificadas para serem apresentadas com o JavaDoc do código. Para auxiliar no uso das anotações, disponibilizamos um tutorial junto ao Framework. Futuramente, é possível desenvolver algum ferramental de apoio para apoiar o uso das anotações.

Apresentaremos o conjunto de anotações já revisado a partir de [Moura and Leite 2020] e a forma de utilizar as anotações. Nosso framework possui anotações para representar elementos (@Actor, @Goal, @Softgoal, @Resource e @Task) e relacionamentos entre os elementos (@Contribution, @TaskDecomposition, @ MeansEnd). A mudança realizada foi a adição de novos atributos nas anotações. Os atributos obrigatórios de cada anotação serão indicados com *.

Para ilustrar o uso das anotações, utilizamos um sistema didático para cadastro de receitas cuja estrutura do projeto encontra-se na Figura 1. A classe ReceitaGui da Figura 1 é a interface gráfica para cadastro das receitas. É a partir dela que os usuários começam a ter suas metas satisfeitas. A classe Receita da Figura 1. É a classe de entidade que será persistida com os dados das receitas, enquanto a classe ReceitaControle trata as solicitações vindas da interface gráfica. Para implementar a persistência das receitas na base de dados, a classe FabricaConexao instancia a conexão com o banco de dados e a classe ReceitaDAO (que implementa a interface IDAO) implementa os métodos com comandos de DML (Data Modification Language).

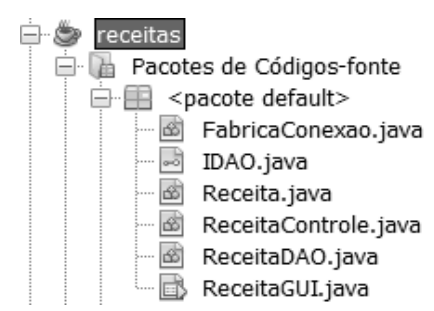

Figura 1. Estrutura do projeto de exemplo "receitas"

Como o modelo de metas é um modelo mais abstrato que o nível de código, o engenheiro de software deve anotar somente as classes e elementos que contribuem para a compreensão das razões da existência aplicação e suas qualidades. Classes e outros elementos cuja razão de ser seja puramente técnica, como FabricaConexao e IDAO, não devem ser anotados. Assim, o modelo ficará mais abstrato e com menos elementos do que o código. Sugerimos que os engenheiros comecem a anotar pelas classes que fazem 
interface com os usuários e utilize o seu conhecimento tácito sobre as razões da aplicação.

@Actor( name*="nome do ator" e type*=[ general | agente | role | position]) - Esta anotação pode ser utilizada em classe, interface (incluindo anotações), ou enumeração. Ela é utilizada para indicar cada ator que está sendo representado por determinado código. É possível utilizar mais de uma @Actor por unidade de código. Ao exportar um modelo $i^{*}$ em qualquer formato, somente os elementos associados a algum ator aparecerão no modelo.

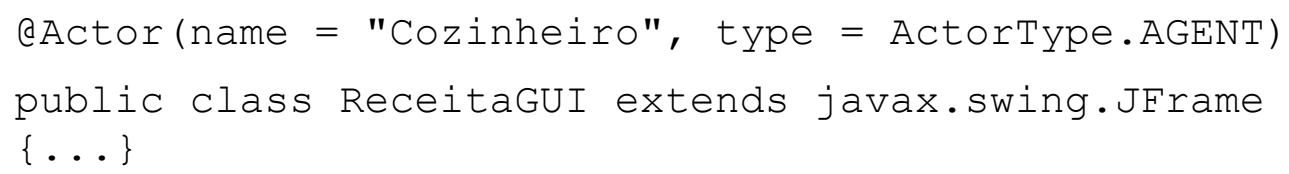

(a)Goal( name*="nome da meta", description="descrição da meta", actor*= "nome do ator") - Esta anotação pode ser utilizada em classe, interface (incluindo anotações) e enumeração para indicar as metas que os atores conseguem e/ou devem alcançar naquela unidade de código. É possível utilizar mais de uma@Goal por unidade de código.

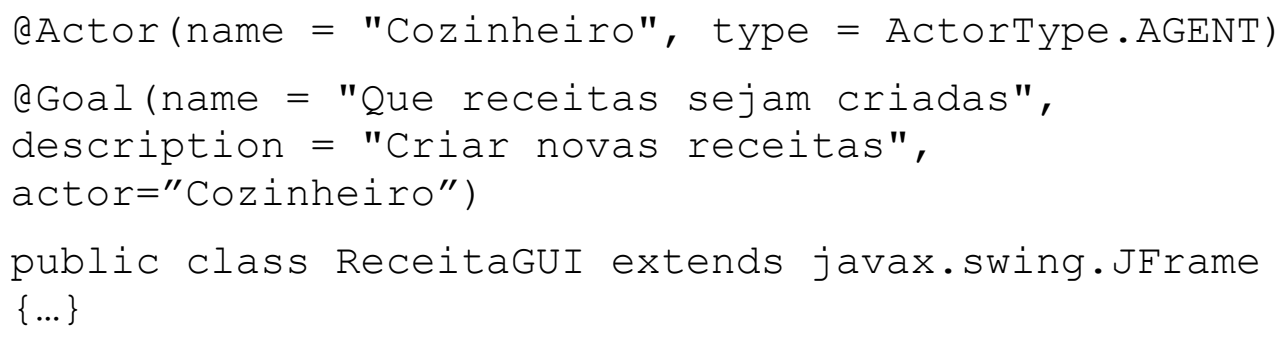

@Softgoal( name*="nome da meta flexível", topic="tópico", description="descrição da meta flexível", elementType $=[\quad$ NFR SOFTGOAL OPERATIONALIZING_SOFTGOAL | CLAIM_SOFTGOAL), label=[ SA-TISFICED | DENIED | CONFLICTING | UNDEFINED], priority=[ CRITI-CAL | DOMINANT | UNDEFINED], actor*="nome do ator") - Esta anotação pode ser utilizada em classe, interface (incluindo anotações), enumeração e/ou atributo para relacionar as metas flexíveis (metas de qualidade) que os atores conseguem e/ou devem alcançar naquela unidade de código. É possível utilizar mais de uma@Softgoal por unidade de código. As operacionalizações destas metas flexíveis, quando disponíveis, serão indicadas pela anotação@Contribution. Nos atributos não obrigatórios que possuem opções prédefinidas, nós destacamos em sublinhado o valor padrão. É importante destacar que esta anotação foi enriquecida com novos atributos característicos do NFR framework [Chung et al. 2000; Supakkul et al. 2010] vislumbrando a possibilidade de criar softgoal interdependency graphs (SIG) dos requisitos não funcionais presentes no sistema. 
(Actor (name="Cozinheiro", type=ActorType.AGENT)

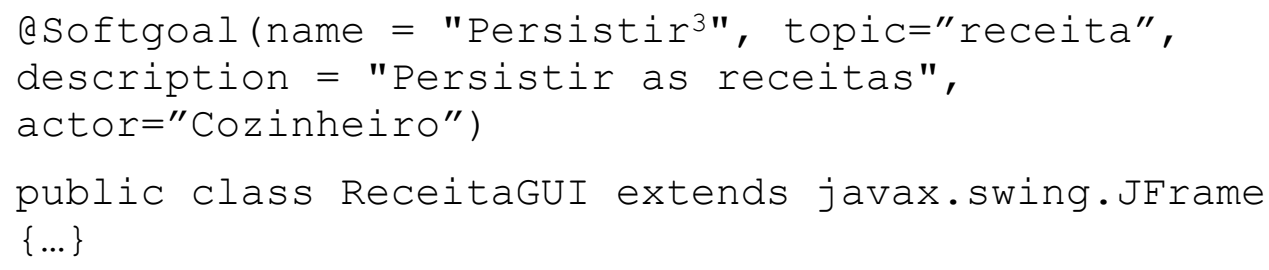

@Task( name*="nome da tarefa", description*="descrição da tarefa" , actor*= "nome do ator") - Esta anotação pode ser utilizada em métodos para fornecer informações acerca da tarefa realizada pelo método em questão.

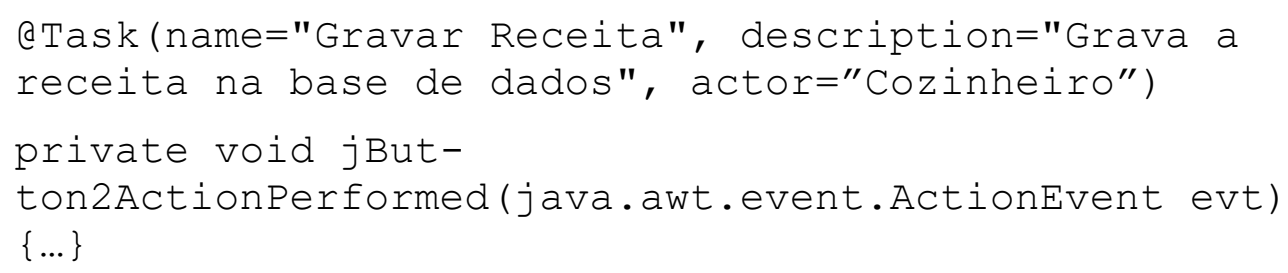

(a) Resource( name*="nome do recurso", actor= "nome do ator") - Esta anotação pode ser utilizada em tipos (i.e.: classe, interface (incluindo anotações), enumeração), atributo e/ou variável local para indicar recursos. É importante ressaltar que uma unidade de código como uma classe pode ser entendida como um recurso, como classes de entidade, por exemplo. No caso de uso em tipos, estes serão apresentados no modelo quando forem utilizados por algum ator e não poderão acumular a anotação @Actor.

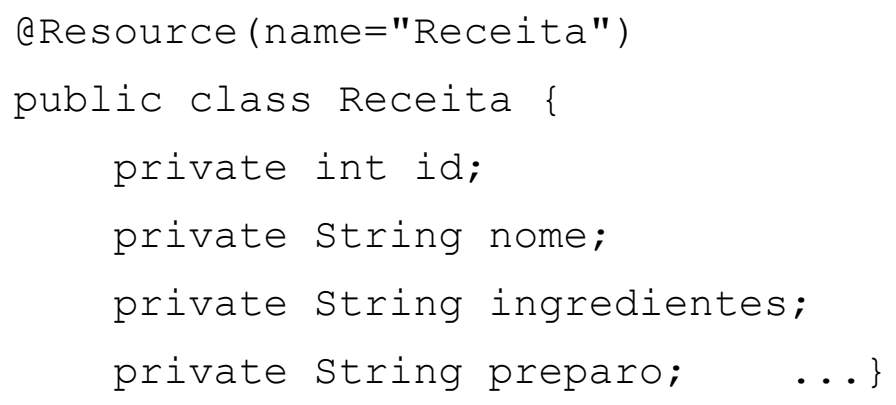

@Contribution( type*=[make | help | hurt | break), softgoal*="meta flexível") - Esta anotação pode ser utilizada em métodos para indicar um relacionamento de contribuição do método para uma meta flexível. Se o método possuir a anotação@Task, esta será relacionada à meta flexível em questão. Caso contrário, será criada uma tarefa com o nome do método. É possível utilizar mais de uma@Contribution por unidade de código.

\footnotetext{
${ }^{3}$ A Persistência é um requisito não funcional que pode ser operacionalizado de diferentes formas como persistência em base de dados ou persistência em arquivo [Silva 2006].
} 


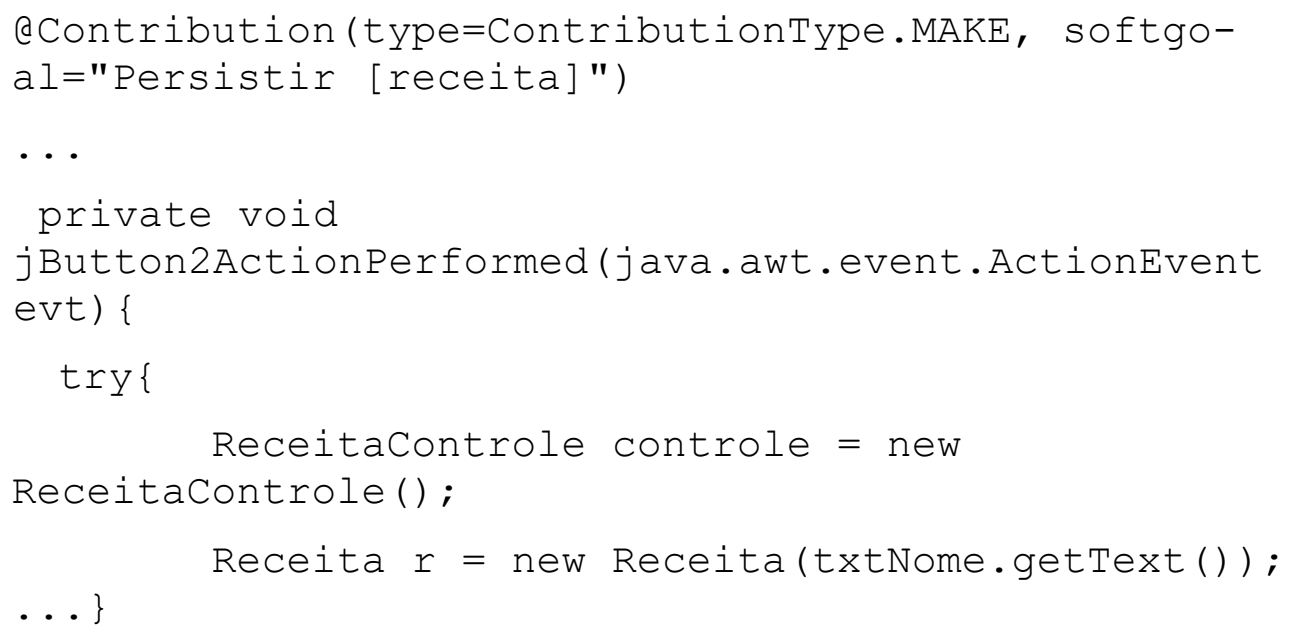

@TaskDecomposition(type*=[resource_for | softgoal_for | goal | sub_task], elemento*="nome do elemento") - Esta anotação pode ser utilizada em métodos, uso de algum tipo ou declaração de parâmetro. Ela indica um relacionamento de decomposição de uma tarefa para uma subtarefa, um recurso para a tarefa, uma submeta ou uma meta flexível para a tarefa. É possível utilizar mais de uma@TaskDecomposition por unidade de código.

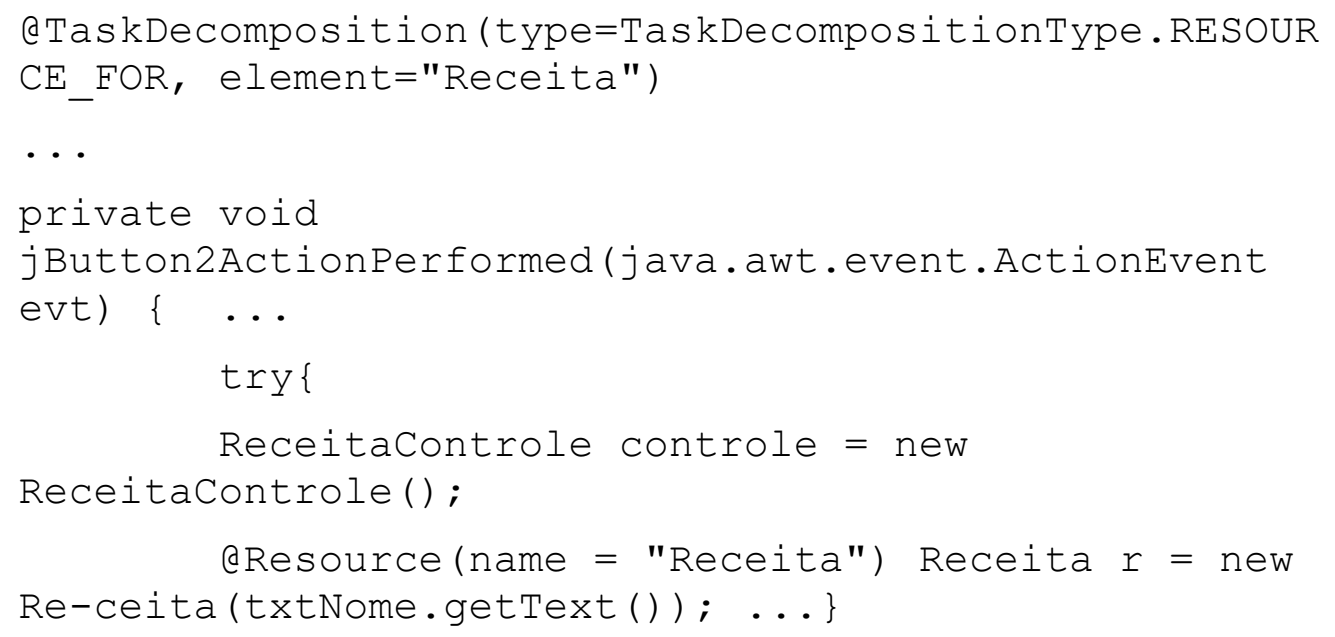

@ MeansEnd( endType*=[resource | goal], end*="nome da meta ou recurso") - Esta anotação pode ser utilizada em métodos para indicar que aquele método é uma tarefa (meio) para alcançar determinada meta, meta flexível ou recurso. É possível utilizar mais de uma@MeansEnd por unidade de código. 


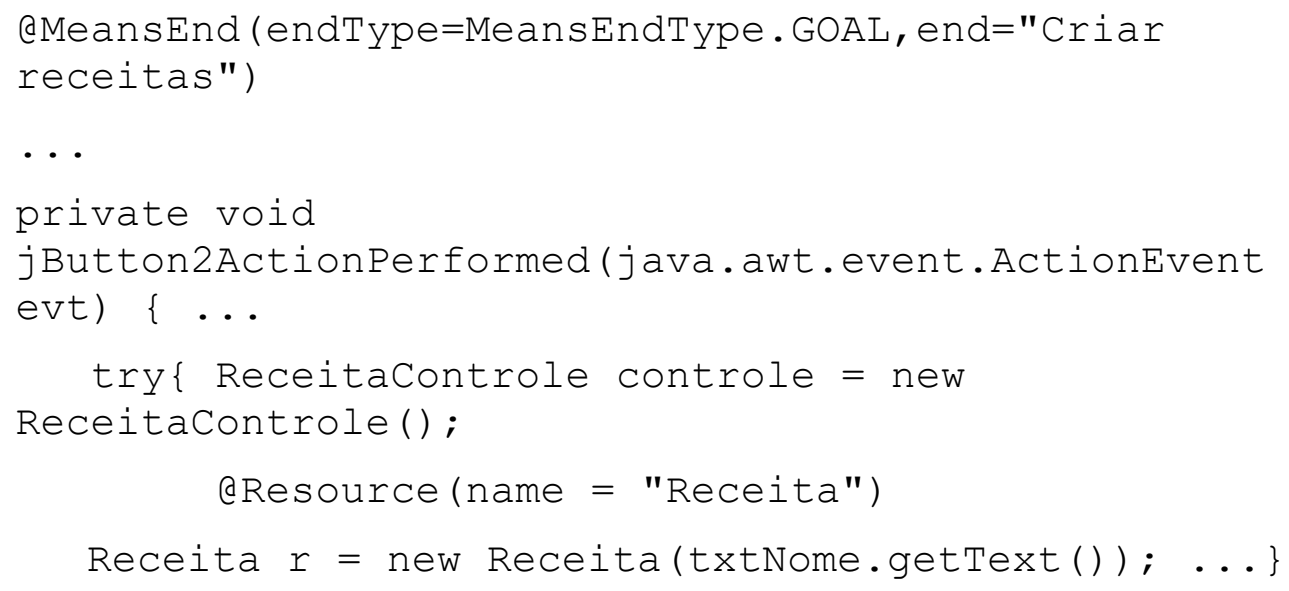

Em futuras versões, desenvolveremos anotações para fornecer meta informações referente aos relacionamentos entre os atores, incluindo informações de dependência. Além disso, estamos averiguando os benefícios de usar a APT (Annotation Processing Tool) para customizar a forma como o compilador Java processa as nossas anotações para o compilador sinalizar erros de uso das anotações em tempo de compilação.

\section{JiStar - Exportador de Modelo i*}

O framework JiStar permite a exportação das metainformações referentes a intencionalidade do código para um modelo $i^{*}$ SR no formato HTML PiStar 2.0 [Pimentel and Castro 2018] e iStarML [Cares et al. 2007]. Quando criarmos anotações para o relacionamento de dependência, poderemos exportar modelos i* SD também. Um modelo de metas facilita a leitura das metas por parte dos engenheiros de requisitos, clientes e todas as partes interessadas no negócio suportado pelo sistema. Com um modelo de metas atualizado em mãos, todas as partes interessadas podem contribuir para a evolução do software.

O exportador de modelos pode gerar modelos de metas $i^{*}$ com base nas seguintes estratégias:

-auto: Gera o modelo de metas utilizando nosso conjunto de heurísticas de código OO para modelos de metas $i^{*}$.

-semiauto: Gera o modelo de metas utilizando nosso conjunto de heurísticas de código OO para modelos de metas i* [Moura 2017] em conjunto com as anotações presentes no código fonte. Nesta estratégia, a anotação tem prioridade sobre as heurísticas.

-manual: Gera o modelo de metas considerando somente as anotações. Esta estratégia é utilizada como padrão quando nenhuma estratégia é informada.

Para gerar o modelo de metas no modo "-manual" a partir do código fonte anotado, após engenheiro de software anotar o código (vide exemplos da Seção 4), deve executar o gerador de modelo de metas passando o caminho dos arquivos compilados do projeto (*.class) e, opcionalmente, o tipo de saída desejada (-html, -pistar ou istarml) e a estratégia de geração de modelo (-auto, -semiauto ou -manual), conforme trecho de comando abaixo (exemplo para Windows). Quando o tipo de saída não é informado, o tipo de saída padrão é o HTML. Quando a estratégia de geração do modelo não é informada, a estratégia padrão é -manual. Neste exemplo, o JiStar gera um arquivo chamado goal_model.txt que pode ser carregado na ferramenta PiStar 2.0. O 
modelo resultante do exemplo é apresentado na Figura 2. O agente Cozinheiro possui a meta "Que receitas sejam criadas" e a meta flexível "Persistir as receitas". A tarefa "Gravar Receita" é um meio para alcançar a meta "Que receitas sejam criadas" e contribui para a meta flexível "Persistir as receitas", para isso, ela consome o recurso "Receita".

$$
\text { java -jar Jistar-1.0.jar "\\caminho\*.class" -pistar }
$$

Neste exemplo da Figura 2, exportamos para o formato PiStar ${ }^{4} 2.0$, que suporta a versão 2.0 do framework i*. Caso tivéssemos usado um relacionamento meios-fim para indicar que a tarefa "Gravar Receita" é o meio para alcança um recurso receita, este não seria mapeado para PiStar e não aparecia no modelo pois o framework iStar 2.0 não possui relacionamento para indicar o meio para alcançar um recurso.

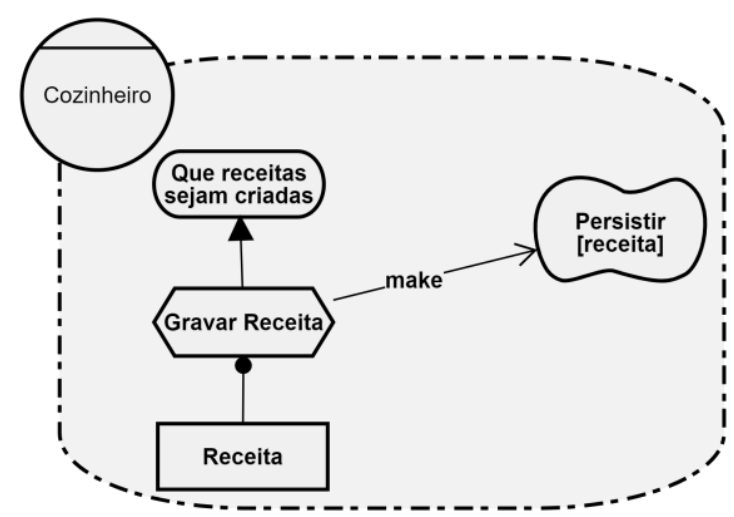

Figura 2. Modelo de metas $i^{\star}$ do exemplo didático sistema de receitas ( PiStar).

No exemplo a seguir, fazemos a exportação do mesmo conjunto de anotações para o formato iStarML que é compatível com o $i^{*} 1.0$. Este comando gera um arquivo com o nome goal_model.istarml que importamos na ferramenta OpenOME ${ }^{5}$ e, como resultado, obtemos o modelo da Figura 3.

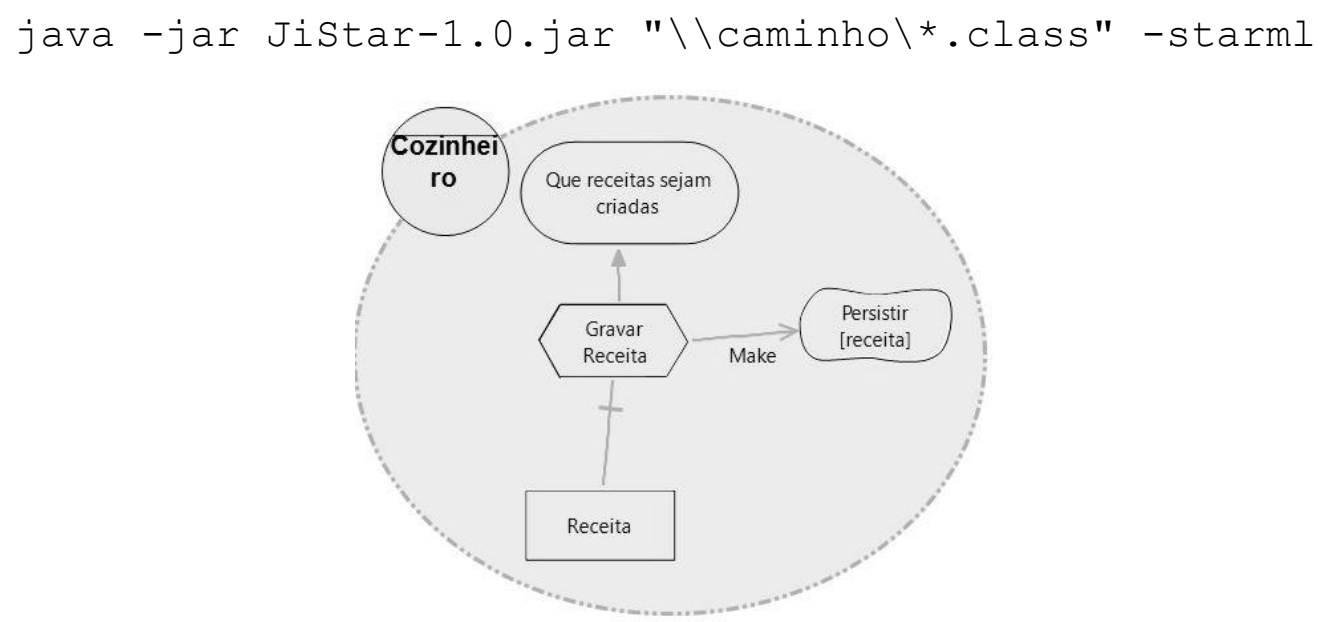

Figura 3. Modelo de metas $i^{\star}$ do exemplo didático sistema de receitas (OpenOME).

\footnotetext{
${ }^{4}$ https://www.cin.ufpe.br/ jhcp/pistar/tool/index.html

${ }^{5} \mathrm{https}: / /$ se.cs.toronto.edu/trac/ome/
} 


\section{Estudo de Viabilidade}

Neste trabalho, relembramos a avaliação da viabilidade do framework JiStar realizada em [Moura and Leite 2020], onde optamos por resgatar as metas do aplicativo RioBus ${ }^{6}$. O RioBus é app desenvolvido em Java para a plataforma Android que mostra a localização geográfica dos ônibus da cidade do Rio de Janeiro. Os dados são oferecidos publicamente pela prefeitura do Rio de Janeiro, em parceria com a FETRANSPOR e Iplanrio. As posições dos ônibus são recuperadas pelo GPS embarcado neles, enviados para a FETRANSPOR e, por fim, a Iplanrio a disponibiliza na página do projeto de dados abertos Data Rio.

\subsection{Estudo}

Preparação do estudo: fizemos o download do código fonte no GitHub, estudamos o código fonte com seus comentários e utilizamos o app.

Execução: realizamos a anotação do código fonte e, para auxiliar a identificação de metas flexíveis (qualidades) de consciência, utilizamos o catálogo de consciência de software [Cunha 2014]. Em seguida fizemos a extração do modelo i* (ver Figura. 4).

Análise do Resultado: o modelo resultante apresenta três atores, onde o ator "Usuário" foi obtido a partir da classe MainActivity, o ator "Marcador de Mapa" foi obtido a partir da classe MapMarker e o ator "Localizador de ônibus" foi obtido a partir da classe BusSearchTask. Os principais elementos internos de cada agente, que são relacionados diretamente ao negócio, foram anotados e o modelo resultante é apresentado na Figura 4. As demais classes não foram consideradas es-enciais para o entendimento do negócio ou foram anotadas como recurso (estas aparecem somente dentro do raciocínio estratégico de cada agente quando anotadas também como um recurso do agente).

Avaliação do Resultado: para avaliar o modelo i* exportado pelo framework JiStar, convidamos três engenheiros de software da indústria com mais de 14 anos de experiência para avaliar o modelo resultante quanto a sua compreensibilidade e usabilidade. A Tabela 2 apresenta as perguntas da avaliação e as respostas dos engenheiros de software.

Ameaças à validade do estudo: o estudo ainda é preliminar e envolveu apenas 3 engenheiros de softwares da indústria. Além disso, as anotações foram aplicadas apenas pelos pesquisadores e os engenheiros avaliaram o resultado, o qual pode variar de acordo com a pessoa que anota o código. $\mathrm{O}$ uso demasiado das anotações pode gerar um modelo com muitos elementos e pouco abstrato. Nesse sentido recomendamos o uso de heurísticas voltadas para minimizar essa prática.

\footnotetext{
${ }^{6}$ https://github.com/RioBus/android-app
} 


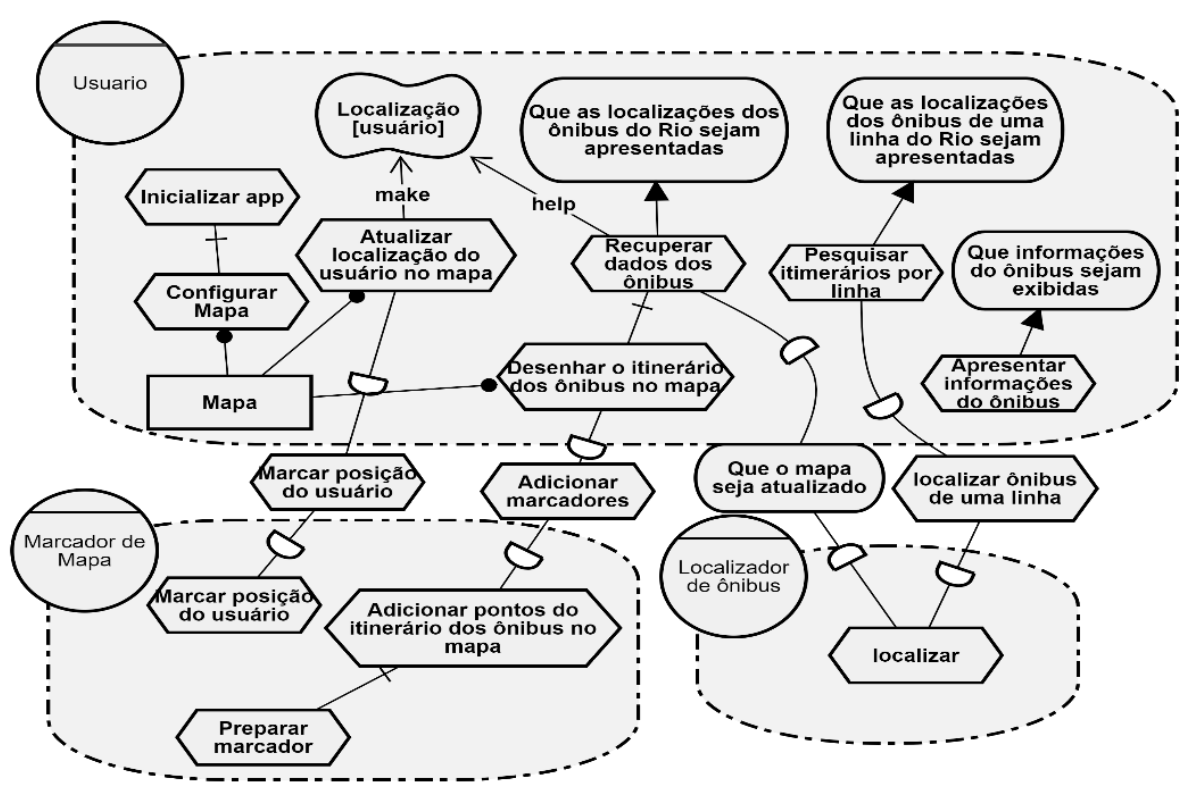

Figura 4. Modelo de metas $\mathrm{i}^{\star}$ do exemplo didático sistema de receitas.

\section{Tabela 2. Perguntas e respostas de avaliação do estudo com engenheiros de software.}

\begin{tabular}{|c|c|c|c|}
\hline Perguntas & $\begin{array}{l}\text { Engenheiro } \\
\text { SW } 1\end{array}$ & $\begin{array}{l}\text { Engenheiro } \\
\text { SW } 2\end{array}$ & $\begin{array}{l}\text { Engenheiro } \\
\text { SW } 3\end{array}$ \\
\hline $\begin{array}{l}\text { Você utilizaria o modelo em reuniões com o } \\
\text { cliente para elicitação de requisitos em uma } \\
\text { evolução do sistema? }\end{array}$ & Sim & $\begin{array}{l}\text { Não, pois os } \\
\text { meus clientes } \\
\text { não conhecem } \\
\text { a linguagem. }\end{array}$ & Sim \\
\hline $\begin{array}{l}\text { Você considera o modelo mais abstrato que o } \\
\text { código? }\end{array}$ & Sim & Sim & Sim \\
\hline $\begin{array}{l}\text { Você conseguiria explicar o propósito do } \\
\text { sistema após ler o modelo? }\end{array}$ & Sim & Sim & Sim \\
\hline $\begin{array}{l}\text { Você considera importante poder manter a } \\
\text { rastreabilidade entre o código e as metas do } \\
\text { sistema? }\end{array}$ & Sim & Sim & Sim \\
\hline
\end{tabular}

Apesar das ameaças à validade do estudo, de acordo com o resultado da avaliação, temos um primeiro indício de que o uso do framework JiStar pode auxiliar na reconciliação de sistemas $\mathrm{OO}$ com suas metas, possibilitando a exportação de um modelo mais abstrato e com linguagem mais apropriada para engenheiros de requisitos e clientes.

\section{Conclusão}

Neste trabalho, propomos o framework JiStar para auxiliar na rastreabilidade para trás entre código fonte $\mathrm{OO}$ e modelos de metas $i^{*}$. Nosso framework contribui para: (A) fornecer um modelo de rastreabilidade do código para as metas pois com o uso das anotações é possível adicionar meta dados de intencionalidade (i.e.: metas) ao código, fazendo a manutenção do vínculo diretamente e somente no código. Quando desejar ver no formato de modelo, basta exportar o modelo; (B) automatizar a exportação de um modelo de metas pois a parir do código anotado com o framework JiStar podemos 
exportar modelos $i^{*}$ nos formatos HTML e PiStar 2.0; (C) prover modelos $i^{*}$ mais abstratos que o código pois seguindo as dicas de uso, os engenheiros de software anotarão somente os elementos do código que possuem relação com a intencionalidade dos usuários (i.e.: suas metas). Isto gera modelos mais abstratos e menos extensos. $\mathrm{O}$ uso demasiado das anotações pode levar a um modelo menos abstrato. e; (D) prover modelos com informações compreensíveis por engenheiros de requisitos e clientes, visto que as anotações permitem o uso do conceito de linguagem da aplicação [Leite and Franco 1990]. Dessa forma, o modelo resultante permite que os engenheiros de requisitos e os clientes se comuniquem e utilizem o modelo para a evolução do software. O engenheiro de software, por sua vez, consegue identificar os pontos de evolução no código devido ao vínculo entre o código e as metas.

Geramos um modelo de metas a partir de sistemas construídos sem a perspectiva GORE, de tal modo que este modelo possua uma linguagem mais apropriada para engenheiros de requisitos e clientes sem perder a rastreabilidade para o código foi alcançado. O modelo resultante pode ser utilizado para a evolução do software. Nossa pesquisa utiliza este modelo na reengenharia de sistemas autoadaptativos.

Futuramente, iremos ampliar nosso conjunto de anotações para contemplar relacionamentos entre atores, incluindo o relacionamento de dependência. Isto permitirá a geração de modelo i* SD. Além disso, pretendemos exportar o modelo também para o formato iStarML [Cares et al. 2007] que suporta o framework i* na versão 1,0 e nos possibilitará gerar diagramas com todas as metainformações que estão disponíveis no JiStar. Realizaremos novos estudos de caso com vistas a avaliar o esforço e a complexidade de uso do framework pois neste trabalho avaliamos o modelo resultante.

\section{Agradecimentos}

Os autores agradecem a Petrobras (Moura) e ao CNPq (Leite) o apoio fornecido para a realização desta pesquisa.

\section{Referências}

Alencar, F. M., Pedroza, F. P., Castro, J. and Amorim, R. C. (2003). New Mechanism for the Integration of Organizational Requirements and Object Oriented Modeling. In WER.

Almentero, E. K. (2013). Dos Requisitos ao Código: Um Processo para Desenvolvimento de Software mais Transparente. PUC-Rio.

Bolchini, D. and Paolini, P. (2002). Capturing Web Application Requirements through Goal-Oriented Analysis. In WER.

Bresciani, P., Perini, A., Giorgini, P., Giunchiglia, F. and Mylopoulos, J. (2004). Tropos: An agent-oriented software development methodology. Autonomous Agents and Multi-Agent Systems, v. 8, n. 3, p. 203-236.

Cares, C., Franch Gutiérrez, J., Perini, A. and Susi, A. (2007). iStarML: The i* mark-up language. 
Castro, J., Alencar, F. and Cysneiros, G. (2000). Closing the gap between organizational requirements and object oriented modeling. Journal of the Brazilian Computer Society, v. 7, n. 1, p. 05-16.

Castro, J., Alencar, F., Santander, V. and Silva, C. (2011). Integration of $i^{*}$ and objectoriented models. Social modeling for requirements engineering, p. 457-484.

Chung, L., Nixon, B. A., Yu, E. and Mylopoulos, J. (2000). Softgoal interdependency graphs. Non-Functional Requirements in Software Engineering. Springer. p. 47-88.

Cunha, H. de S. (2014). Desenvolvimento de Software Consciente com Base em Requisitos. PUC-Rio.

Dalpiaz, F., Franch, X. and Horkoff, J. (2016a). istar 2.0 language guide. arXiv preprint arXiv:1605.07767,

Dalpiaz, F., Franch, X. and Horkoff, J. (17 nov 2016b). iStar tutorial online. https://www.dropbox.com/s/412k4tbywb8wekk/iStar-tutorial-online.pdf?dl=0, [accessed on Mar 27].

Darwish, N. R. and Zohdy, B. S. (2016). Goal Modeling Techniques for Requirements Engineering. International Journal of Computer Science and Information Security, v. 14, n. 7, p. 739.

Gonçalves, E., Heineck, T., Araújo, J. and Castro, J. (2018). A Catalogue of iStar Extensions. In WER.

Horkoff, J., Li, T., Li, F.-L., et al. (2015). Using goal models downstream: a systematic roadmap and literature review. International Journal of Information System Modeling and Design (IJISMD), v. 6, n. 2, p. 1-42.

Jaqueira, A., Lucena, M., Alencar, F. M., Castro, J. and Aranha, E. (2013). Using i* Models to Enrich User Stories. iStar, v. 13, p. 55-60.

Leite, J. and Franco, A. P. M. (1990). O uso de hipertexto na elicitação de linguagens da aplicação. Anais de IV Simpósio Brasilero de Engenharia de Software, SBC, p. 134149.

Malta, Á., Soares, M., Santos, E., et al. (2011). iStarTool: Modeling Requirements using the i* Framework. In IStar.

Moura, A. M. da M. (2017). Awareness Driven Software Reengineering. In Requirements Engineering Conference (RE), 2017 IEEE 25th International. . IEEE. 
Moura, A. M. da M., De Oliveira, R. F., Fernandes, E., et al. (2019). Improving Urban Mobility for the Visually Impaired using the Awareness Quality. In Proceedings of the XVIII Brazilian Symposium on Software Quality.

Moura, A. M. da M. and Leite, J. C. S. do P. (2020). JiStar - Rastreabilidade Entre Código Java e Modelos de Metas i*. . http://wer.inf.pucrio.br/WERpapers/artigos/artigos_WER20/15_WER_2020_paper_25.pdf.

Oliveira, R. F. De, Moura, A. M. da M. and Leite, J. C. S. P. (2018). Reengineering for Accessibility: A Strategy Based on Software Awareness. In Proceedings of the 17th Brazilian Symposium on Software Quality. . ACM.

Pimentel, J. and Castro, J. (2018). pistar tool-a pluggable online tool for goal modeling. In 2018 IEEE 26th International Requirements Engineering Conference (RE). . IEEE.

Sayão, M. and Do Prado Leite, J. C. S. (2006). Rastreabilidade de requisitos. RITA, v. 13 , n. 1, p. 57-86.

Serrano, M. (2011). Desenvolvimento Intencional de Software Transparente Baseado em Argumentação. PUC-Rio.

Serrano, M. and Leite, J. C. S. do P. (2011). Development of Agent-Driven Systems: from $i^{*}$ Architectural Models to Intentional Agents Code. In iStar. . Citeseer.

Silva, L. F. Da (2006). Uma Estratégia Orientada a Aspectos para a Modelagem de Requisitos. Tese de Doutorado, Computer Science Department, PUC-Rio.

Silva, L. F. Da, Leite, J. C. do P. and Breitman, K. K. (2004). C \& L: uma ferramenta de apoio à engenharia de requisitos. PUC.

Supakkul, S., Hill, T., Chung, L., Tun, T. T. and Do Prado Leite, J. C. S. (2010). An NFR pattern approach to dealing with NFRs. In Requirements Engineering Conference (RE), 2010 18th IEEE International. . IEEE.

Yu, E. (1995). Modelling strategic relationships for process reengineering.

Yu, E. (2001). Agent orientation as a modelling paradigm. Wirtschaftsinformatik, v. 43, n. 2, p. 123-132.

Yu, Y., Wang, Y., Mylopoulos, J., et al. (2005). Reverse engineering goal models from legacy code. In Requirements Engineering, 2005. Proceedings. 13th IEEE International Conference on. . IEEE. 